LETTER TO THE EDITOR

\title{
A new approach to detect coherent modes using microwave reflectometry
}

\author{
T Estrada ${ }^{1}$, T. Happel ${ }^{2}$ and E. Blanco ${ }^{1}$ \\ ${ }^{1}$ Laboratorio Nacional de Fusión. Euratom-CIEMAT Association, 28040 \\ Madrid, Spain \\ ${ }^{2}$ Max-Planck-Institut für Plasmaphysik, Euratom-IPP Association, 85748 \\ Garching, Germany \\ E-mail: teresa.estrada@ciemat.es
}

\begin{abstract}
.
A new approach to detect coherent modes using microwave reflectometry is presented. The proposed approach is based on the modulation of the reflecting layer in the poloidal direction due to the presence of rotating coherent MHD modes. The applicability of the approach is demonstrated, using either conventional or Doppler reflectometry, in plasmas with high background turbulence level where the standard analysis method fails.
\end{abstract}

PACS numbers: 52.70.Gw, 52.55.-s, 52.35.Bj, 52.35.Ra

\section{Introduction}

Microwave reflectometry technique has experienced significant advances in the last two decades becoming a very attractive diagnostic presently used in almost all fusion devices. This technique allows measuring electron density profiles, plasma instabilities, turbulence and radial electric fields with excellent spatial and temporal resolution. Conventional reflectometry has been applied to turbulence measurements since the 1980's, although in some cases, the reflecting layer for microwave frequencies shows a complex corrugated structure which can lead to strong interference in the reflected beam that hinders the density fluctuation information and makes difficult to extract quantitative information. Even in those cases, valuable qualitative information on the turbulence characteristics can be obtained as, for example, in turbulence studies during the development of transport barriers (e.g. [?, ?, ?]). Quantitative description of the density fluctuations is possible for specific scenarios and in particular for coherent plasma perturbations, e.g. MHD modes [?] or high frequency Alfven eigenmodes [?, ?, ?]. Both, radial localization and amplitude characterization of the modes are possible provided their intensity is high enough to be detected above the background turbulence. Doppler reflectometry makes use of a finite tilt angle between the probing beam and the cut-off layer normal to measure the Bragg back-scattered process that takes place at the cut-off layer. This technique allows the measurement of the density turbulence and its perpendicular rotation velocity, at different turbulence scales and with good spatial and temporal resolution [?, ?, ?, ?, ?]. Besides, coherent MHD and/or Alfven modes may also be visible in the Doppler reflectometry signals. 
The present letter reports on a new approach to detect coherent modes using microwave reflectometry. Results are presented showing the applicability of the approach even in plasmas with high turbulence level where the standard method fails. The approach consists on the application of a specific analysis method to the standard complex amplitude signal, $A e^{i \phi}$ ( $A$ and $\phi$ being the amplitude and the phase of the signal reflected at the plasma cut-off layer), measured with reflectometry, either conventional or Doppler. The method is described in section 2 and its application to reflectometry signals obtained in the TJ-II stellarator using both, conventional and Doppler reflectometers is shown in section 3. Finally, a brief discussion is included in section 4.

odigjt

\section{Description of the method}

The proposed approach is based on the modulation of the reflecting layer in the perpendicular or poloidal direction due to presence of coherent MHD or Alfven modes. As the modes rotate in the perpendicular direction, the modulation produces a periodic change in the effective angle between the probing beam and the cut-off layer normal, $\theta$. This is schematically represented in figure ??. In Doppler reflectometry the change in the angle appears as a modulation in the Doppler peak frequency: $f_{D}=u_{\perp} k_{\perp} / 2 \pi$, with $k_{\perp}=2 k_{0} \sin \theta, k_{0}$ being the probing beam wavenumber and $u_{\perp}$ the perpendicular rotation velocity. In conventional reflectometry, a small misalignment between the probing beam and the cut-off layer normal produces an asymmetry in the $A \mathrm{e}^{i \phi}$ spectrum as it results from a combination of the unshifted reflected wave and the Doppler shifted backscattered one. As a consequence, periodic change in the angle appears as a modulation in the degree of asymmetry in the spectrum which gives rise to a modulation in the so-called phase-runaway. In both cases, the Doppler peak frequency or the mean frequency of the complex amplitude spectrum appears modulated at the frequency of the coherent mode.

In general, to accurately determine the center of gravity of the spectrum (a good representative of either the Dopler peak frequency or the degree of asymmetry in the spectrum) rather long time intervals with high enough sampling frequencies are needed. However, as it will be shown below, extremely short time intervals with only few samples are enough to monitor the changes in the center of gravity, COG, linked to rotating coherent modes. As a result, it is possible to track the evolution of the COG with high time resolution and consequently discriminate coherent modes even at frequencies in the range of the Alfven eigenmodes, i.e. hundreds of $\mathrm{kHz}$. The method owes its interest to the fact that the COG is affected mainly by changes in the probing angle, but not by changes in the plasma turbulence. Consequently, coherent modes can be detected regardless the plasma turbulence level.

\section{Application of the method}

In order to demonstrate the applicability of the method, we have selected plasma discharges of the TJ-II stellarator. Older discharges in which reflectometry measurements were carried out using a conventional system with standard horn antennas [?], and more recent discharges in which an optimized Doppler reflectometer front-end was operative [?]. In both cases, fast frequency hopping systems with heterodyne detection are used at the reflectometer back-end [?]. 

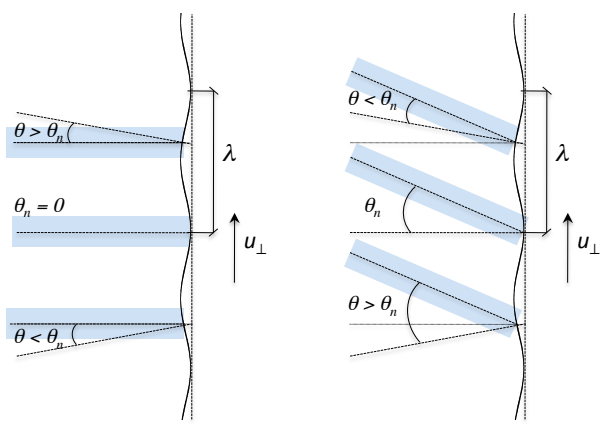

Figure 1. Schematic representation of the modulation of the angle between the probing beam (represented by a blue shadow area) and the plasma cut-off layer normal due to a rotating coherent mode in both, conventional (left) and Doppler reflectometry (right). As the mode rotates at $u_{\perp}$, the probing angle $\theta$ is modulated around the nominal value $\theta_{n}$.

\subsection{Conventional reflectometry}

Conventional reflectometers working either at fixed frequency or in frequency hopping mode provide information on both, broadband turbulence and coherent modes. In TJ-II a conventional reflectometer was in operation until December 2008 [?]. In-phase $(I=A \cos \phi)$ and quadrature $(Q=A \sin \phi)$ signals were recorded at 5 $\mathrm{MHz}$ sampling rate during the entire discharge duration. An example is shown in figure ??. Figure ??.a shows the spectrogram of the complex amplitude signal, $A \mathrm{e}^{i \phi}$, measured during the NBI phase of the plasma discharge, where high frequency Alfven eigenmodes are destabilized [?]. To calculate the spectrogram, time realizations of $204.8 \mu$ s are considered, i.e. each spectrum is calculated using 1024 samples of the $I$ and $Q$ signals. In this figure, the frequency range has been limited to $\pm 500 \mathrm{kHz}$ to highlight the coherent mode detected at about $200 \mathrm{KHz}$. Although the mode is visible, its amplitude is very close to the background broadband turbulence level. Figure ??.b shows the spectrogram of the COG of the $A \mathrm{e}^{i \phi}$ spectra, while figure ??.c shows the COG time evolution; only a very short time interval, $0.2 \mathrm{~ms}$, is shown in figure ??.c to mark off the coherent oscillation. The COG is calculated every $1.6 \mu$ s, i.e., only 8 samples of the $I$ and $Q$ signals recorded at $5 \mathrm{MHz}$ are sufficient to track the $\mathrm{COG}$ of the $A \mathrm{e}^{i \phi}$ spectrum; as a result, frequencies up to $300 \mathrm{KHz}$ can be measured as shown in figure ??.b. The coherent modes at frequencies of about 150 and $200 \mathrm{kHz}$ are more clearly visible in the spectrogram of the COG, figure ??.b, than in the spectrogram of the complex amplitude $A \mathrm{e}^{i \phi}$, figure ??.a, because the COG is affected by the coherent modes but not by the background broadband turbulence.

As it has been already pointed out, to accurately determine the COG of the spectrum rather long time intervals are needed whereas short time intervals with only few samples are enough to monitor COG oscillations linked to rotating coherent modes. To illustrate this point a one millisecond time window of shot 18730 has been selected and both, the mean value of the COG and the COG spectrum are calculated for an increasing number of samples. The result is shown in figure ??. The 1 ms time window with $N$ number of points, is divided in intervals of $2^{n}$ points, with $n=1$, 2, etc. For each interval, $i$, the FFT of $A \mathrm{e}^{i \phi}$ is calculated and from the spectrum, $S_{i}(f)$, the COG is calculated as: $C O G_{i}=\sum_{k=1}^{2^{n}} f_{k} S_{i}\left(f_{k}\right) / \sum_{k=1}^{2^{n}} S_{i}\left(f_{k}\right)$. Its 

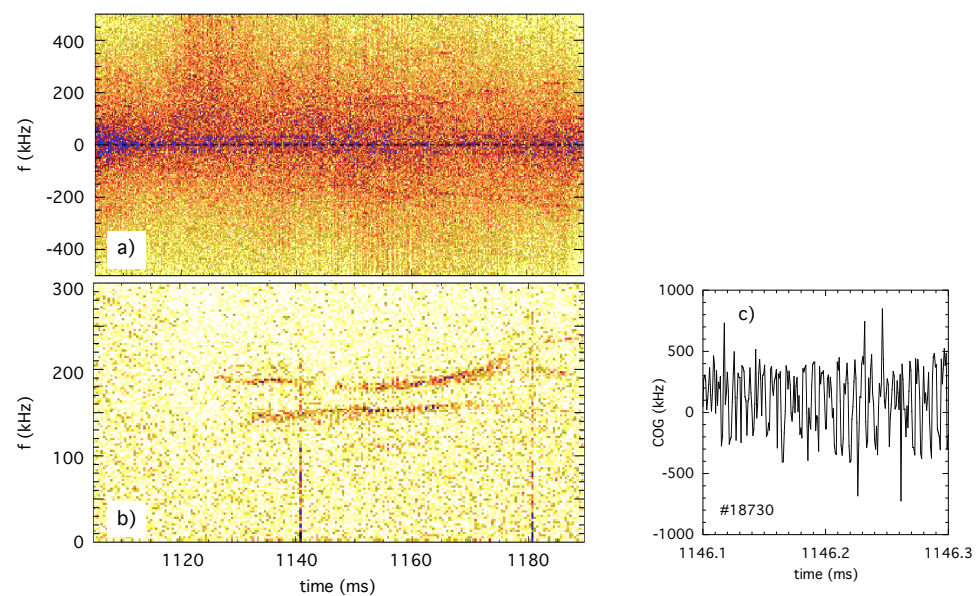

Figure 2. (a): Spectrogram of the complex amplitude signal, $A e^{i \phi}$, measured by the conventional reflectometer in the shot 18730. (b) Spectrogram of the center of gravity calculated using the same data as in (a). The time evolution of the center of gravity during a a very short time window of $0.2 \mathrm{~ms}$ is shown in (c). Note that the vertical lines at $t=1140$ and $1180 \mathrm{~ms}$ in (b) are related to instrumental effects.

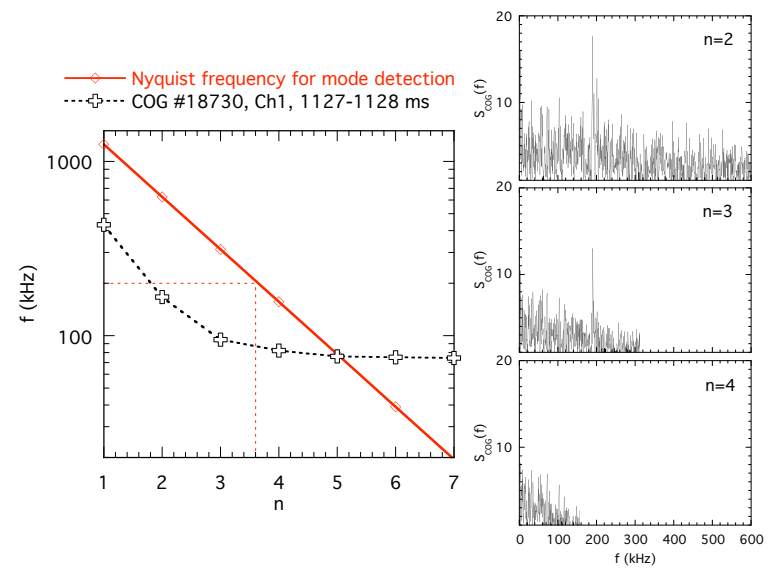

Figure 3. Left: COG calculated considering time intervals with $2^{n}$ samples as a function of $n$ (black crosses) and the resulting Nyquist frequency of the COG spectrum (red diamonds). Right: COG spectra for $n=2,3$ and 4.

mean value, $\mathrm{COG}=m^{-1} \sum_{i=1}^{m} C O G_{i}, m$ being the number of intervals $m=N / 2^{n}$, is shown in figure ??.left and its spectrum, $S_{C O G}$, in figure ??.right for $n=2$, 3 and 4 . As the number of samples increases, the frequency resolution improves and the COG tends to the right value, reached for $n>5$. At these values, the COG time resolution is poor and therefore the Nyquist frequency of $S_{C O G}$ is low preventing the detection of high frequency modes. This example shows that although the error in the determination of the COG can be high if short time intervals are considered, its temporal variation contains valuable information for the detection of 
coherent modes. As it has been already described, in conventional reflectometry the recorded $A \mathrm{e}^{i \phi}$ signal is a combination of the unshifted reflected wave and the Doppler shifted backscattered one. The unshifted reflected wave has a symmetric spectrum, i.e. the COG is zero and is not affected by the rotating mode, only the amplitude of the spectrum may be affected. However, the frequency of the Doppler shifted backscattered wave is modified following the modulation of $\theta$ and produces the modulation in the COG of the $A \mathrm{e}^{i \phi}$ spectrum. Therefore, although the spectrum calculated at each time interval, $S_{i}$, is only meaningful if enough number of samples are considered to calculated it, the changes in its shape due to changes in the frequency of the Doppler shifted component can be followed even taking an extremely small amount of samples.

Conventional reflectometry measurements are often devoted to spatially localize coherent MHD or Alfven modes. However, the mode identification is only possible provided its intensity is high enough to be detected above the background turbulence. Often, this is not an issue when measuring well at the plasma interior, however, close to the plasma edge, plasma turbulence can be high preventing mode detection. The example shown in figure ?? has been selected to illustrate this point. In this case, the conventional reflectometer was operated in frequency hopping mode to measure at different radial positions: the frequency was stepped from 33 to $49 \mathrm{GHz}$ in 10 steps within $50 \mathrm{~ms}$. Figure ??.a shows the spectrogram of the complex amplitude $A \mathrm{e}^{i \phi}$, measured during the NBI phase of the discharge. The radial position of the cut-off layer for each probing frequency is written in the upper part of the figure. In this case, to calculate the spectrogram time realizations of $102.4 \mu \mathrm{s}$ are considered, i.e. each spectrum is calculated using 512 samples of the $I$ and $Q$ signals. A coherent mode of about $200 \mathrm{kHz}$ is detected at inner radial positions, $\rho<0.4$, but not further outside, what might be - erroneously- interpreted as a consequence of the localization of the mode at the plasma core. However, the analysis of the COG yields a different conclusion. The spectrogram of the COG is shown in figure ??.b. The mode is clearly visible not only at the plasma core but also at outer radial positions up to $\rho \approx 0.7$. As before, the COG is calculated every $1.6 \mu$ s, i.e., only 8 samples of the $I$ and $Q$ signals recorded at $5 \mathrm{MHz}$ are enough to track the COG of the $A \mathrm{e}^{i \phi}$ spectrum. Figures ??.c and ??.d show the spectrum of the COG measured at $\rho=0.68$ and $\rho=0.38$, respectively. The amplitude of the mode decreases towards the plasma edge what prevents its detection using the standard analysis, i.e. the spectrogram of the complex amplitude signal. It is worth mentioning that the coherent mode detection using the spectral analysis of the reflectometer phase, $\phi$, proposed in [?], yields similar results as those found using the spectral analysis of the $A \mathrm{e}^{i \phi}$. As in figure ??.a, in the spectrogram of $\phi$, the background broadband turbulence prevents the mode detection in the radial range $\rho>0.4$.

As it has been previously reported in [?], the coherent mode shown in figure ?? corresponds to an $m=5$ Helical Alfvén Eigenmode (HAE). In that work, the radial localization of the mode was experimentally studied through the correlation between Mirnov coil signals and Heavy Ion Beam Probe (HIBP) measurements radially localized with a resolution of $\approx 1 \mathrm{~cm}$. It was shown that the $m=5$ mode has a rather broad radial extension, in good agreement with the result obtained using the COG approach. However, it has to be noted that the radial localization of coherent modes using reflectometry can also be affected by small-angle scattering processes taking place along the wave propagation path. These processes have been theoretically studied in [?] and their effect on the reported measurements cannot be, in principle, 


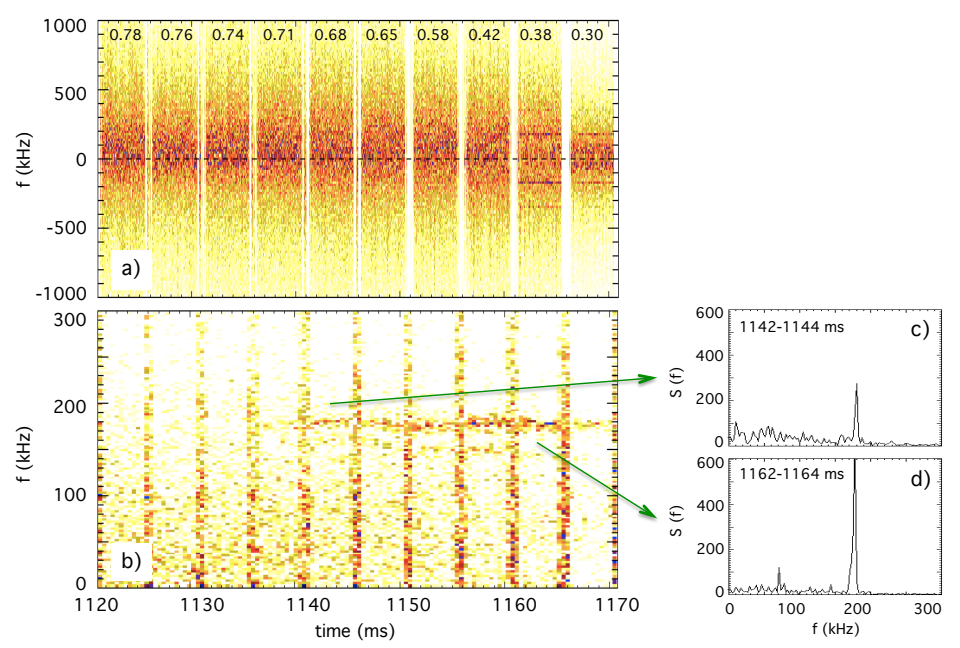

Figure 4. (a): Spectrogram of the complex amplitude signal, $A e^{i \phi}$, measured by the conventional reflectometer in the shot 15570 , working in hopping mode between 33 and $49 \mathrm{GHz}$ : the probing frequency is stepped every $5 \mathrm{~ms}$; the corresponding cut-off layer radial positions are indicated for each probing frequency. (b) Spectrogram of the center of gravity calculated using the same data as in (a). (c) and (d) Spectrum of the center of gravity, averaged during a 2 ms time window, measured at $\rho=0.68$ and 0.38 , respectively

ruled out. According to these studies, a coherent mode localized in a certain plasma region, e.g. in $\rho \approx 0.7$, can be also detected when measuring at inner radial positions. The signal amplitude, in these cases, is reported to be lower than that measured when the mode is located at the cut-off layer position. In our experiments, the COG approach reveals a radial variation of the mode intensity, being stronger at inner radial locations. This radial dependence could be used as a hint in favor of the good localization of the measurements. Although out of the scope of this letter, it is worth mentioning that an analytical treatment, similar to the one reported in [?], could allow a more quantitative analysis of the reflectometry data.

\subsection{Doppler reflectometry}

Doppler reflectometry is used to measure the density turbulence and its perpendicular rotation velocity, at different turbulence scales and with good spatial and temporal resolution [?, ?, ?, ?]. In TJ-II, an optimized Doppler reflectometer is in operation since 2009 [?, ?]. Although coherent MHD and/or Alfven modes are sometimes visible in the Doppler reflectometry signals, in most cases the spectrogram of the complex amplitude does not show coherent peaks. However, as it will be illustrated in this section, the COG calculated with high time resolution can be used to track these modes. The Doppler reflectometer $I$ and $Q$ signals are recorded at $10 \mathrm{MHz}$ sampling rate. Therefore, using the same number of points to calculate the COG as in the conventional reflectometer measurements, frequencies of up to $600 \mathrm{kHz}$ can be detected. Alternatively, double number of samples can be used (16 instead of 8) improving the determination of the COG while keeping the frequency range up to 300 

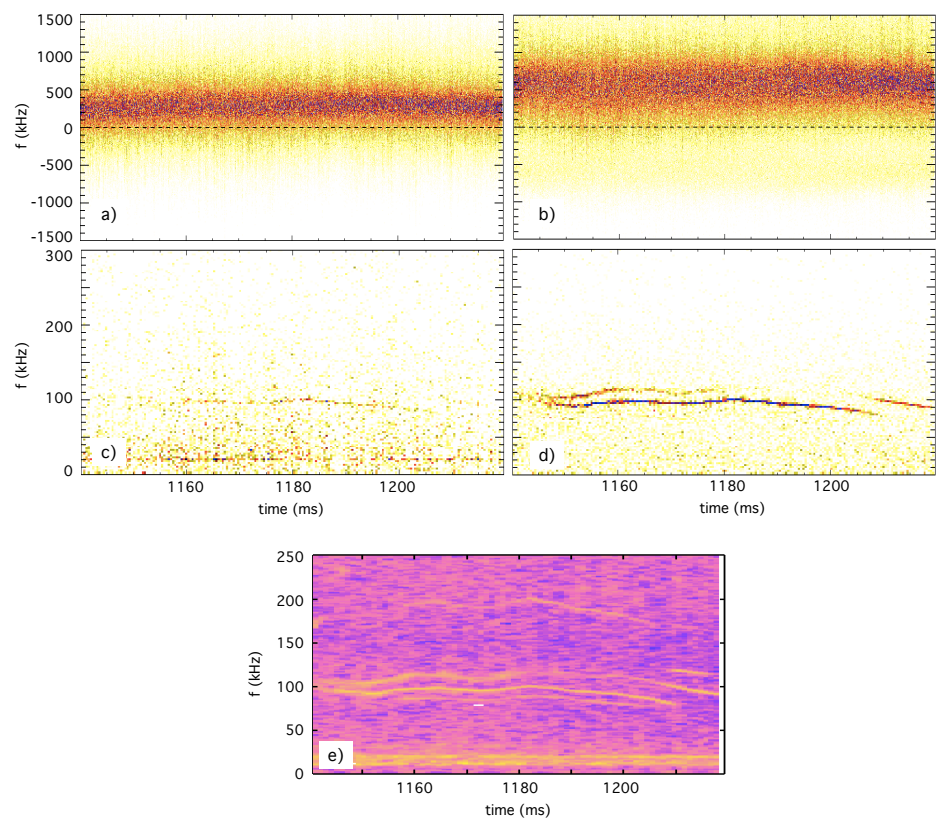

Figure 5. (a, b): Spectrograms of the complex amplitude signals, $A e^{i \phi}$, measured by the Doppler reflectometer in the shot 30094 at (a) $f_{1}=33 \mathrm{GHz}\left(\rho_{1} \approx 0.85\right)$ and (b) $f_{2}=40 \mathrm{GHz}\left(\rho_{2} \approx 0.7\right)$. (c, d) Spectrograms of the center of gravity calculated using the same data as in (a, b). (e) Spectrum of a Mirnov coil signal.

$\mathrm{kHz}$.

An example is shown in figure ??. The top panels show the spectrogram of the complex amplitude $A \mathrm{e}^{i \phi}$, measured using two channels working at frequencies $f_{1}=33$ $\mathrm{GHz}$ (figure ??.a) and $f_{2}=40 \mathrm{GHz}$ (figure ??.b), simultaneously. The spectrograms are calculated using time realizations of $102.4 \mu \mathrm{s}$, i.e. each spectrum is calculated using 1024 samples of the $I$ and $Q$ signals. In the spectrograms, only the Doppler peak is visible with a Doppler frequency of about $f_{D_{1}} \approx 300 \mathrm{kHz}$ in the first channel and $f_{D_{2}} \approx 600 \mathrm{kHz}$ in the second one. The spectrograms of the respective COG are shown in figures ??.c and ??.d and the spectrogram of a Mirnov coil signal is plotted in figure figure ??.e. In this case, the COG is calculated every $1.6 \mu$ s, i.e., using 16 samples of the $I$ and $Q$ signals recorded at $10 \mathrm{MHz}$. Multiple coherent modes are detected by the Mirnov coils, some of them also present in the COG spectrograms. The inner channel $\left(\rho_{2} \approx 0.7\right)$ detects a rather intense mode at about $100 \mathrm{kHz}$ and a lighter one at slightly higher frequency (figure ??.d). However, the $100 \mathrm{kHz}$ mode is only weakly detected by the outer channel $\left(\rho_{2} \approx 0.85\right)$ where a lower frequency mode of about $20 \mathrm{kHz}$ becomes visible (figure ??.c).

As for the conventional reflectometry case, the COG of the Doppler reflectometry signals of shot 30094 has been calculated using an increasing number of samples. The procedure applied to calculate the COG and the COG spectra shown in figure ?? is applied here. The result is shown in figure ??. The COG is shown in figure ??.left and its spectrum, $S_{C O G}$ for $n=2,5$ and 6 in figure ??.right. As the number of samples increases, the frequency resolution of the $A \mathrm{e}^{i \phi}$ spectrum improves and the COG tends 


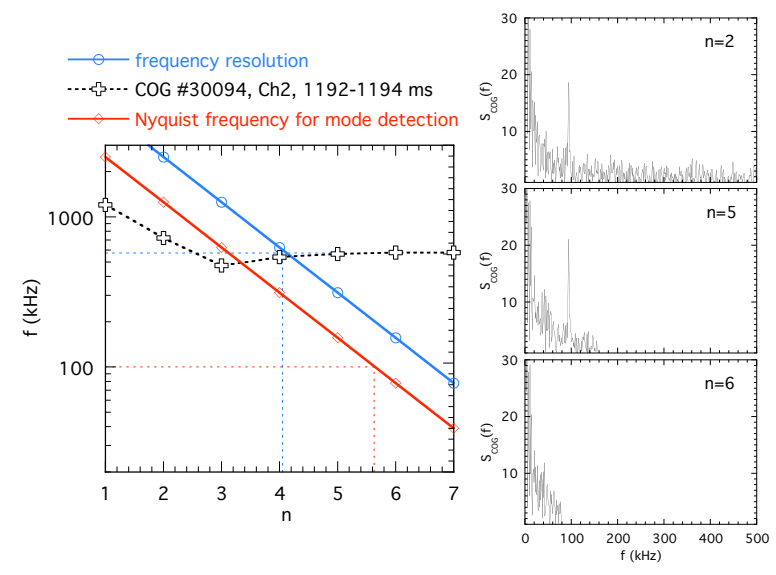

Figure 6. Left: COG calculated considering time intervals with $2^{n}$ samples as a function of $n$ (black crosses), the corresponding frequency resolution of the $A \mathrm{e}^{i \phi}$ spectrum (blue circles) and the resulting Nyquist frequency of the COG spectrum (red diamonds). Right: COG spectra for $n=2,5$ and 6 .

to the right value, reached for $n \geq 4$. It is worth to note that a frequency resolution in the $A \mathrm{e}^{i \phi}$ spectrum close to the Doppler frequency seems to be enough to obtain the later. In this example, for $n=4$ and 5, the COG time resolution is still good enough, the Nyquist frequency of $S_{C O G}$ being 312 and $156 \mathrm{kHz}$, respectively, to allow both, a precise determination of the COG and the detection of the modes. Shorter time intervals, with $n<4$, could be considered if coherent modes with $f>312 \mathrm{kHz}$ are to be detected even though the COG is not accurately determined. For longer time intervals, $n \geq 6$, the COG is properly determined but the coherent modes frequency (of about $100 \mathrm{kHz}$ ) lies above the $S_{C O G}$ Nyquist frequency.

This example shows how Doppler reflectometry measurements can be used to localize coherent MHD or Alfven modes. Furthermore, as the Doppler reflectometer measures the perpendicular rotation velocity of the plasma turbulence, information on the mode characteristics, poloidal mode number and wavelength, can be inferred. Assuming that the mode rotates as the plasma turbulence, the mode wavelength can be calculated as the ratio between rotation velocity and mode frequency, and the poloidal mode number as the ratio between plasma radius and mode wavelength. Finally, qualitative information on the intensity of the coherent mode, i.e. on the degree of modulation in the reflecting layer, can be obtained through the amplitude of the coherent mode in the COG spectra measured for instance using small frequency steps in hopping mode.

\section{Discussion}

The examples discussed in this letter show how coherent rotating modes modulate the COG of the complex amplitude spectra measured using either conventional or Doppler reflectometry diagnostics. The COG is affected mainly by changes in the effective angle between the probing beam and the cut-off layer normal, but not by changes in the plasma turbulence. Consequently, coherent modes can be detected by 
applying the COG approach even extreme cases in which the mode intensity is very low and is masked in the $A \mathrm{e}^{i \phi}$ and $\phi$ spectra by the background turbulence. High intensity modes are in general visible using either the COG approach or the $A \mathrm{e}^{i \phi}$ or $\phi$ spectra. However, occasionally they could also be masked by the turbulence in the $A \mathrm{e}^{i \phi}$ spectrum. If the mode produces a strong modulation in the reflected wave phase, it gives rise to multiple peaks with lower amplitude in the $A \mathrm{e}^{i \phi}$ spectrum which could lie below the background turbulence. In these cases, the $\phi$ spectrum, as well as the COG approach, would provide better results as the mode appears as a single high amplitude peak.

In the examples reported in this letter, the COG is modulated by the change in the effective angle between the probing beam and the cut-off layer normal, due to rotating coherent modes. In Doppler reflectometry, oscillations in the COG, or equivalently in the Doppler peak frequency, can be also interpreted as changes in the turbulence propagation velocity, i.e. in the perpendicular $E \times B$ flow, related for instance to GAMs [?, ?, ?]. The modulation in the Doppler peak frequency and the absence of modulation in the amplitude of the back-scattered signal is argued to be a signature of $E \times B$ flow modulation. However, this might be not always the case. The modulation in the COG due to a rotating coherent mode may be much more intense than that in the signal amplitude. This happens for instance in the example shown in figures ??.b and ??.d. The spectrum of both, COG and amplitude, averaged in the time interval 1180-1185 ms, are shown in figure ??. A rather intense peak at about $f \approx 100 \mathrm{kHz}$, detected in the COG, is absent in the signal amplitude spectrum. The reason may be related to a high turbulence level that prevents the coherent mode detection using the amplitude signal. In fact, measurements performed under the same plasma conditions but at inner radial positions, where the turbulence level is lower, show the modulation in both, COG and amplitude spectrum.
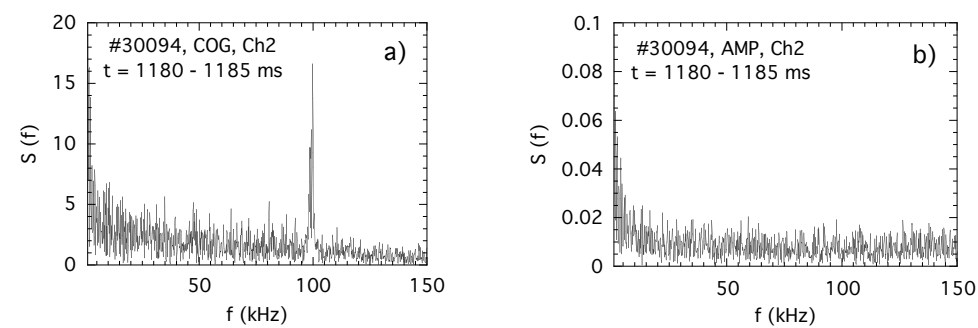

Figure 7. Spectrum of the COG (a) and spectrum of the amplitude (b), measured by the Doppler reflectometer in the shot 30094 during the time intervat 1180-1185 ms, with $f_{2}=40 \mathrm{GHz}\left(\rho_{2} \approx 0.7\right)$.

Therefore, in order to discriminate between rotating MHD modes and oscillations in the $E \times B$ flow, a dual Doppler reflectometry system measuring simultaneously at $\pm \theta$ as those described in [?, ?] is needed. Otherwise, additional information is required as for instance, information on magnetic oscillations for MHD modes or information on plasma temperature to compare the mode frequency with the theoretical GAM dispersion relation $[?, ?]$. 


\section{Acknowledgments}

The authors acknowledge the entire TJ-II team for their support and in particular E. Ascasíbar for Mirnov coils data and B. Ph. van Milligen for fruitful discussions. This work has been partially funded by the Spanish Ministry of Science and Innovation under contract number ENE2010-18409.

\section{References}

[1] K. H. Burrell et al., Plasma Physics and Controlled Fusion 34, 1859 (1992).

[2] G. D. Conway et al., Phys. Rev. Lett. 84, 1463 (2000).

[3] N. Oyama and K. Shinohara, Rev. Sci. Instrum. 73, 1169 (2002).

[4] R. Sabot et al., Plasma Physics and Controlled Fusion 48, B421 (2006).

[5] L. F. Ruchko et al., Rev. Sci. Instrum. 75, 655 (2004).

[6] S. Hacquin et al., Plasma Physics and Controlled Fusion 49, 1371 (2007).

[7] S. da Graça et al., Plasma Physics and Controlled Fusion 49, 1849 (2007).

[8] M. Hirsch et al., Plasma Physics and Controlled Fusion 43, 1641 (2001).

[9] P. Hennequin et al., Rev. Sci. Instrum. 75, 3881 (2004).

[10] G. D. Conway et al., Plasma Physics and Controlled Fusion 46, 951 (2004).

[11] T. Happel et al., Rev. Sci. Instrum. 80, 073502 (2009).

[12] T. Estrada et al., Plasma Phys. Control. Fusion 51, 124015 (2009).

[13] T. Estrada et al., Nucl. Fusion 46, S792 (2006).

[14] L. Cupido, J. Sánchez, and T. Estrada, Rev. Sci. Instrum. 75, 3865 (2004).

[15] R. Jiménez-Gómez et al., Nuclear Fusion 51, 033001 (2011).

[16] J. C. Hillesheim et al., Rev. Sci. Instrum. 81, 10D907 (2010).

[17] E. Z. Gusakov et al., Plasma Physics and Controlled Fusion 44, 1565 (2002).

[18] T. Happel, E. Blanco, and T. Estrada, Rev. Sci. Instrum. 81, 10 D901 (2010).

[19] G. D. Conway et al., Plasma Physics and Controlled Fusion 47, 1165 (2005).

[20] L. Schmitz et al., Rev. Sci. Instrum. 79, 10F113 (2008).

[21] M. Hirsch and E. Holzhauer, Plasma Physics and Controlled Fusion 46, 593 (2004).

[22] M. Hirsch, E. Holzhauer, and H.-J. Hartfuss, Nuclear Fusion 46, S853 (2006). 